\title{
Saturable Absorbers with Concentration-Dependent Absorption Recovery Time
}

\author{
A. Penzkofer
}

Naturwissenschaftliche Fakultät II - Physik, Universität Regensburg, D-8400 Regensburg, Fed. Rep. Germany

Received 28 October 1985/Accepted 22 February 1986

\begin{abstract}
The ground-state bleaching of highly concentrated rhodamine $6 \mathrm{G}$ solutions in methanol is studied with intense picosecond light pulses. The ground-state recovery time changes with concentration from about $3.9 \mathrm{~ns}$ at low concentration $\left(10^{-5} \mathrm{~mol} / \mathrm{dm}^{3}\right)$ to about $1 \mathrm{ps}$ at high concentration $\left(0.6 \mathrm{~mol} / \mathrm{dm}^{3}\right)$. The shortening of the absorption recovery time is determined by the concentration dependent quenching of the $S_{1}$-state population due to unbound dimers and by the intensity dependent longitudinal and transverse amplified spontaneous emission.
\end{abstract}

PACS: $42.65,42.70,42.20$

The bleaching of absorbing media by intense light pulses is a general phenomenon. The only requirement for reduction of absorption with increasing light signal is that the ground-state absorption cross-section is larger than the excited-state absorption cross-section [1-5]. For laser pulse durations $\Delta t_{L}$ short compared to the absorption recovery time $\tau_{A}$ the bleaching behavior is determined by the input pulse energy density $\varepsilon$ [6]. At a pulse energy density $\varepsilon_{S}=h v_{L} / \sigma_{L}$ the ground-state population is reduced to approximately half its initial value (exact value depends on absorption dynamics [6]). $\varepsilon_{S}$ is called the saturation energy density, $v_{L}$ is the laser frequency and $\sigma_{L}$ is the ground-state absorption cross-section. For laser pulse durations long compared to the absorption recovery time the light transmission is intensity dependent. At a pulse intensity of $I_{S}=h v_{L} / \sigma_{L} \tau_{A}$, the saturation intensity, the groundstate population is reduced to approximately half its initial value $[1-6]$.

The application of saturable absorbers as modelocking dyes requires absorption recovery times short compared to the resonator round-trip time. In passively mode-locked $\mathrm{Nd}$ lasers and ruby lasers (small stimulated emission cross-section, no gain dependent pulse shortening) absorption recovery times of some picoseconds are necessary for picosecond pulse generation [7]. Shortening of picosecond pulses to the subpicosecond region may be achieved by passing picosecond pulses through saturable absorbers of some picosecond absorption recovery time [8-10]. The radiative $S_{1}-S_{0}$ lifetime of dyes is generally between 1 and $10 \mathrm{~ns}$. Picosecond absorption recovery times are due to fast radiationless $S_{1}-S_{0}$ relaxation by internal conversion. At low concentrations fast internal conversion is found for molecules with flexible structure in low viscous solutions [11-14]. For infrared dyes the internal conversion is fast because of the small $S_{0}-S_{1}$ energy gap [15-17]. At high concentrations the $S_{1}-S_{0}$ lifetime is shortened by selfquenching [11, 12, 18-21]. Molecular aggregates (dimers, trimers, etc. and molecules in near distance) with fast internal conversion rate are formed. Molecular diffusion and Förster-type energy transfer transport the excitation to the quenching centers and a fast absorption recovery occurs $[11,12,22]$.

In this paper the concentration dependent absorption recovery time of rhodamine $6 \mathrm{G}$ in methanol is studied. Bleaching experiments are carried out at concentrations of $10^{-5}, 10^{-4}, 10^{-3}, 10^{-2}, 0.04,0.1,0.2,0.4$, and $0.6 \mathrm{~mol} / \mathrm{dm}^{3}$ with single picosecond second-harmonic 
light pulses of a mode-locked Nd-glass laser. The absorption recovery time reduces with increasing concentration. At $C=0.6 \mathrm{~mol} / \mathrm{dm}^{3}$ an absorption recovery time of $0.8 \pm 0.3 \mathrm{ps}$ is measured. The reduction of the absorption recovery time is determined by quenching of the $S_{1}$-state lifetime due to unbound dimers [22] and - in an intermediate concentration region - due to longitudinal and transverse amplified spontaneous emission.

\section{Experimental Arrangement}

The bleaching of concentrated solutions of rhodamine $6 \mathrm{G}$ in methanol at room temperature is studied with the experimental setup depicted in Fig. 1. Single picosecond light pulses are selected from a passively modelocked Nd-phosphate glass laser. The pulses are amplified and the second harmonic is generated in a KDP crystal (pulse duration $\Delta t_{L} \simeq 4 \mathrm{ps}$ FWHM). The intensity of the pulses at the sample is varied with filters and a lens [beam diameter at sample $d_{0} \simeq(0.8 \pm 0.2) \mathrm{mm}$ (FWHM)]. At low concentrations the dye is kept in conventional glass cuvettes $(l=2 \mathrm{~cm}$ for $C=10^{-5} \mathrm{~mol} / \mathrm{dm}^{3}, l=2 \mathrm{~mm}$ for $C=10^{-4} \mathrm{~mol} / \mathrm{dm}^{3}$ ). For high concentrations $\left(C \geqq 10^{-3} \mathrm{~mol} / \mathrm{dm}^{3}\right)$ the dye solution is contained in a thin cell of adjustable thickness [22]. Thicknesses between 0 and $200 \mu \mathrm{m}$ are achieved by screw adjustment without spacers. Above $200 \mu \mathrm{m}$ thickness spacers may be inserted into the cell. The energy transmission through the dye cell is measured with photodetectors PD1 and and PD3. The input peak pulse intensity is determined by twophoton transmission measurements through a rutile crystal with photodetectors PD1 and PD2 [23]. For the 0.4 molar rhodamine $6 \mathrm{G}$ solution the temporal behavior of the absorption recovery was studied by measuring the energy transmission of a weak probe pulse versus delay time in a pump and probe arrange-

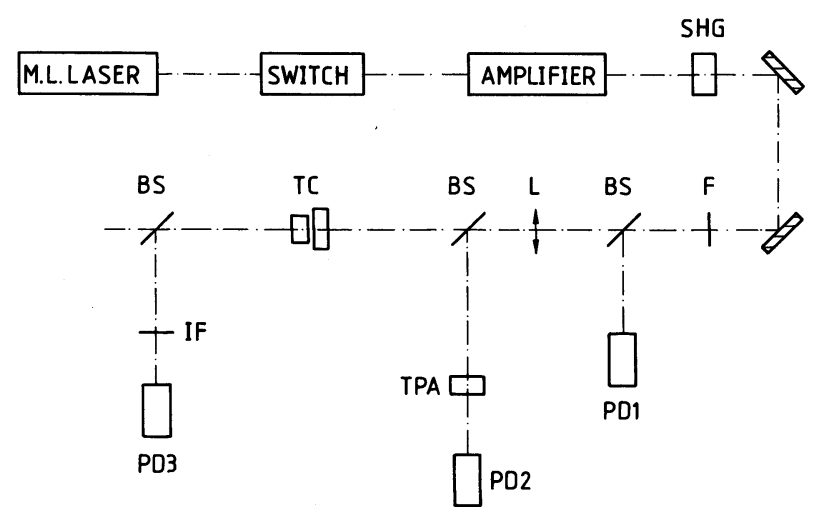

Fig. 1. Experimental setup. SHG: KDP crystal; F: filter; BS: beam splitter; L: lens $(f=50 \mathrm{~cm})$; TC: thin cell of variable thickness; TPA: rutile crystal for intensity detection; PD1-PD3: photodetectors; IF: interference filter) ment. For the 0.04 molar rhodamine $6 \mathrm{G}$ solution the transverse amplified spontaneous emission was detected by replacing the variable thin cell by a standard glass cuvette and measuring the perpendicular fluorescence emission with a photodetector.

\section{Results}

Bleaching experiments were carried out for dye concentrations between $10^{-5}$ and $0.6 \mathrm{~mol} / \mathrm{dm}^{3}$. In Figs. 2 and 3 obtained energy transmission data points are plotted. The depicted curves are calculated by applying the rate equation system of [6] to the level system shown in Fig. 4. The effects of amplified spontaneous emission are not included in these calculations $\left(\sigma_{\mathrm{em}}=0\right.$, $\tau_{v}=0$ ). The used dye parameters are listed in the figure captions. The different absorption cross-sections of monomers and unbound dimers (present at high

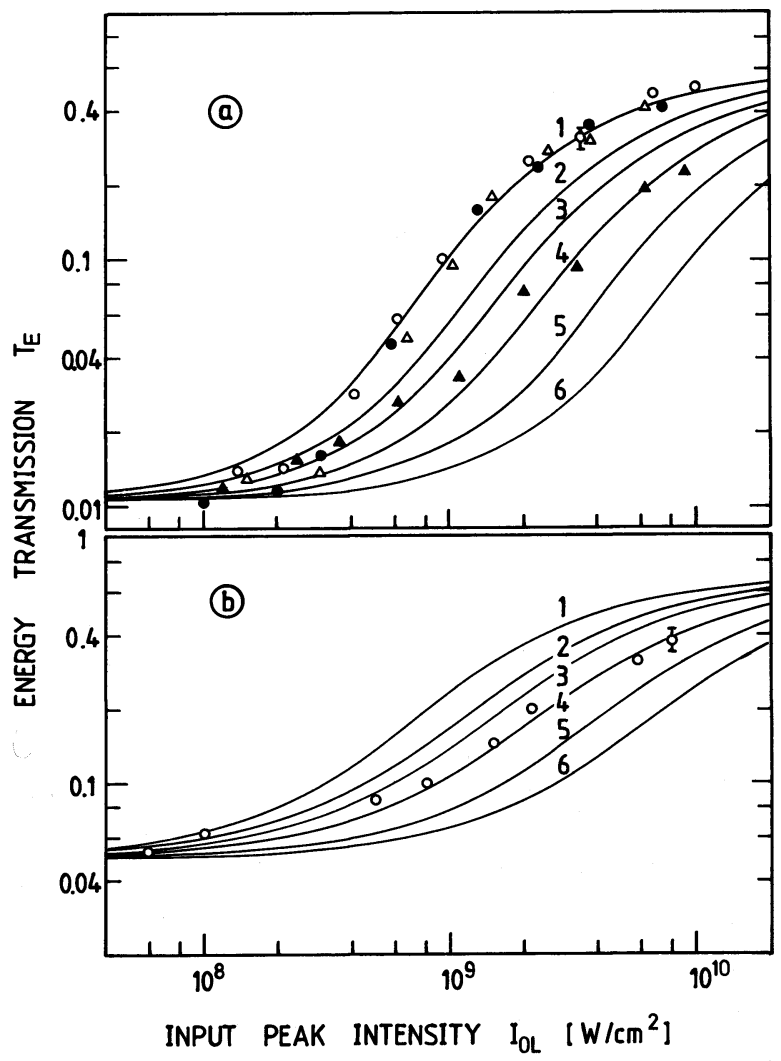

Fig. $2 \mathrm{a}$ and $\mathrm{b}$. Energy transmission through rhodamine $6 \mathrm{G}$ in methanol. Experimental points: (a) Concentrations $C=10^{-5} \mathrm{~mol} / \mathrm{dm}^{3}(0), 10^{-4} \mathrm{~mol} / \mathrm{dm}^{3}(\bullet), 10^{-3} \mathrm{~mol} / \mathrm{dm}^{3}(\triangle)$, and $10^{-2} \mathrm{~mol} / \mathrm{dm}^{3}$ (А). (b) $0.04 \mathrm{~mol} / \mathrm{dm}^{3}$. Calculated curves: (1) $\tau_{F}=3.9 \mathrm{~ns},(2) \tau_{F}=4 \mathrm{ps},(3) \tau_{F}=2 \mathrm{ps},(4) \tau_{F}=1 \mathrm{ps},(5) \tau_{F}=0.5 \mathrm{ps},(6)$ $\tau_{F}=0.25$ ps. Pump pulse duration $\Delta t_{L}=4$ ps. Dye parameters: $\sigma_{\text {ex }, L}=5 \times 10^{-17} \mathrm{~cm}^{2}, \quad \tau_{F C}=0.7 \mathrm{ps} \quad[27], \tau_{\text {ex }}=0.1 \mathrm{ps} \quad[27]$, $\tau_{0 r}=170 \mathrm{ps}$ [34], $\tau_{v}=0, \sigma_{\mathrm{em}}=0$. Special values for (a): $T_{0}=0.01$, $\sigma_{L}=4.05 \times 10^{-16} \mathrm{~cm}^{2}, \quad$ and for (b): $T_{0}=0.05$, $\sigma_{L}=3.75 \times 10^{-16} \mathrm{~cm}^{2}$ 


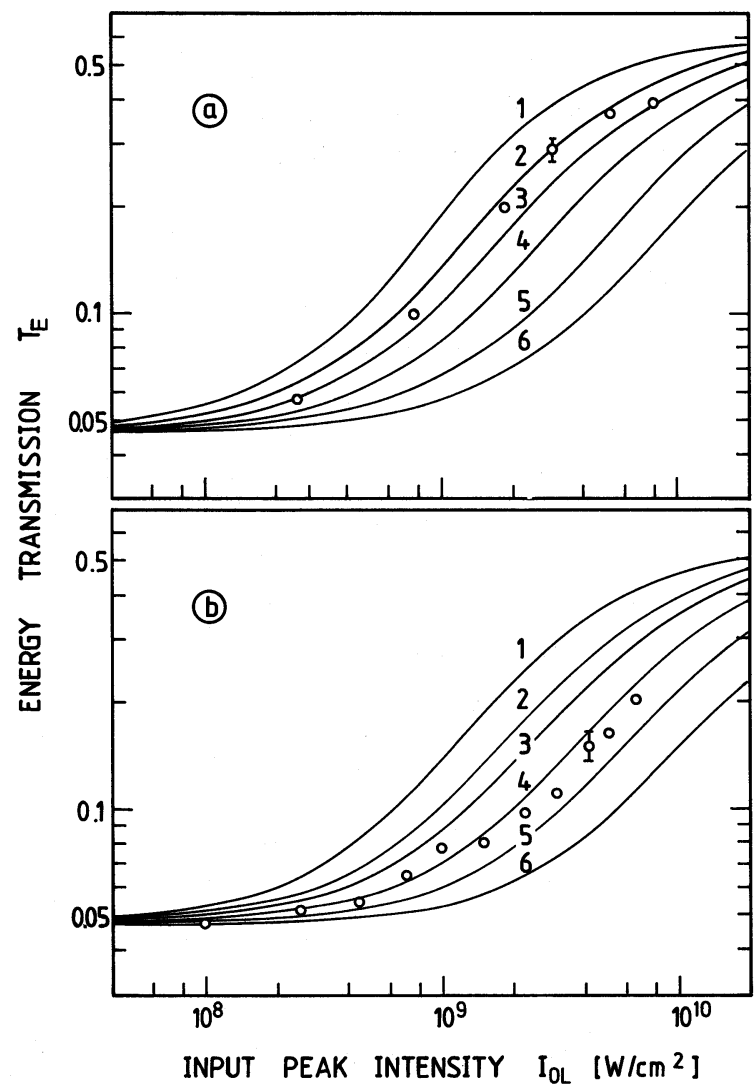

Fig. 3a and b. Energy transmission through rhodamine $6 \mathrm{G}$ in methanol. (a): $\quad C=0.2 \mathrm{~mol} / \mathrm{dm}^{3}, \quad \sigma_{L}=3.06 \times 10^{-16} \mathrm{~cm}^{2}$, $T_{0}=0.046$. (b): $C=0.6 \mathrm{~mol} / \mathrm{dm}^{3}, \sigma_{L}=2.2 \times 10^{-16} \mathrm{~cm}^{2}, T_{0}=0.046$. Other parameters of curves as in Fig. 2

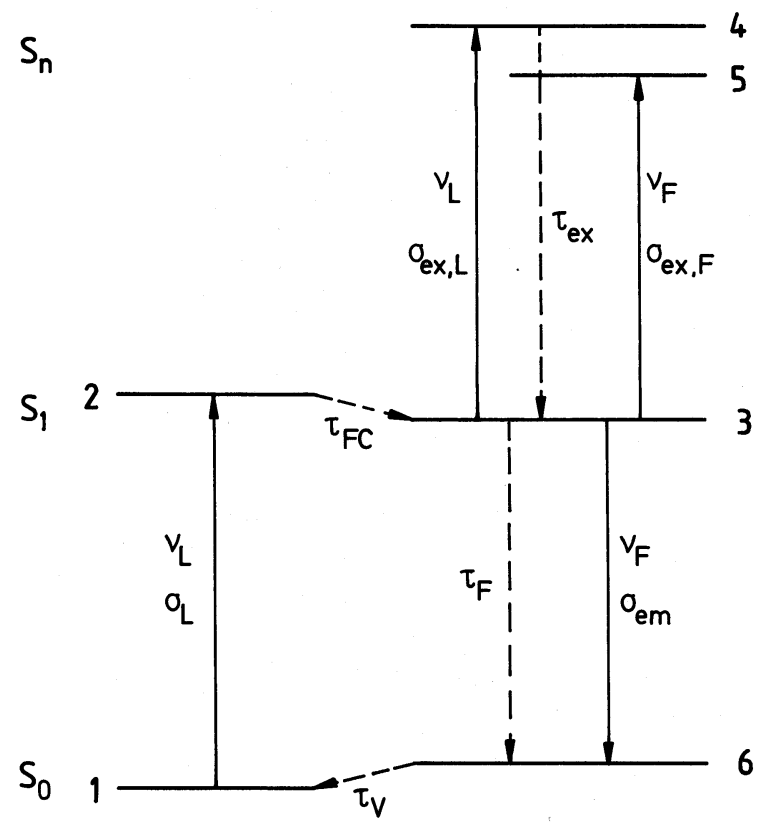

Fig. 4. Level scheme of dye used in calculations concentration [24]), the energy transfer between monomers and dimers and the different lifetimes of monomers and dimers [22] are not separated out. These facts are only included by using a concentration dependent average absorption cross-section $\sigma_{L}=-\ln \left(T_{0}\right) / N_{A} C l\left(T_{0}\right.$ : transmission at concentration $C ; N_{A}$ : Avogadro's constant) and an average recovery time $\tau_{A}$. The average absorption cross-section $\sigma_{L}$ at the laser wavelength, $\lambda_{L}=526.5 \mathrm{~nm}$, is shown in Fig. 5 . The $\sigma_{L}$ values were obtained by transmission measurements through thin cells $\left(C \leqq 0.15 \mathrm{~mol} / \mathrm{dm}^{3}\right)$ and by use of a reflection technique $\left(C>0.15 \mathrm{~mol} / \mathrm{dm}^{3}\right)$ [24].

In Fig. $2 \mathrm{a}$ the experimental points for $C=10^{-5}, 10^{-4}$, and $10^{-3} \mathrm{~mol} / \mathrm{dm}^{3}$ fit to an absorption recovery time $\tau_{A}$ long compared to the pulse duration $\Delta t_{L}$ and an excited state absorption cross section $\sigma_{\text {ex, } L}=5 \times 10^{-17} \mathrm{~cm}^{-2}$ (see also [25]). The actual value of $\tau_{A}$ cannot be determined since for $\tau_{A} \gg \Delta t_{L}$ the energy transmission is only energy dependent (same bleaching curve for all $\tau_{A} \gg \Delta t_{L}$ ). A pump and probe experiment would be necessary to resolve the absorption recovery time for $\tau_{A} \gg \Delta t_{L}$. For $C=0.01 \mathrm{~mol} / \mathrm{dm}^{3}$ the experimental points (full triangles) do not fit to a single energy transmission curve. The absorption recovery time $\tau_{A}$ shortens with increasing pump pulse intensity. This shortening of the absorption recovery time will be explained below by transverse amplified

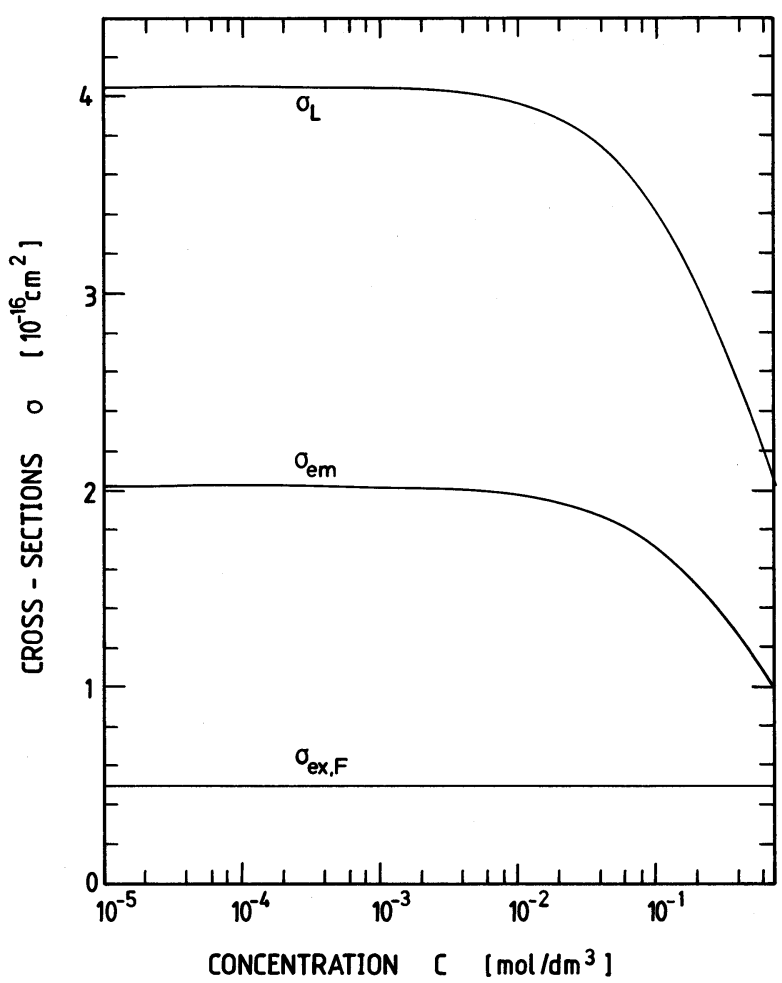

Fig. 5. Concentration dependence of cross-sections. $\sigma_{L}$ from [24], $\sigma_{\mathrm{em}}$ approximated, $\sigma_{\mathrm{ex}, F}$ assumed to be equal to $\sigma_{\mathrm{ex}, L}$ 
spontaneous emission. The same situation is observed for the 0.04 molar (Fig. 2b) and the 0.1 molar (no curve shown) rhodamine $6 \mathrm{G}$ solution. At even higher concentration the experimental energy transmission points again follow a theoretical bleaching curve, i.e. $\tau_{A}$ becomes independent of $I_{0 L}$. In Fig. 3a the situation is depicted for a 0.2 molar solution. An absorption recovery time of $\tau_{A}=(3.5 \pm 1) \mathrm{ps}$ is resolved. In Fig. $3 \mathrm{~b}$ the bleaching of 0.6 molar rhodamine $6 \mathrm{G}$ is illustrated. The comparison of the calculated curves with the experimental points gives an absorption recovery time of $\tau_{A}=0.8 \pm 0.3 \mathrm{ps}$.

For the 0.4 molar rhodamine $6 \mathrm{G}$ solution a pump and probe experiment was performed. The transmission curve versus time was found to be symmetric to the zero-delay point within the experimental accuracy.

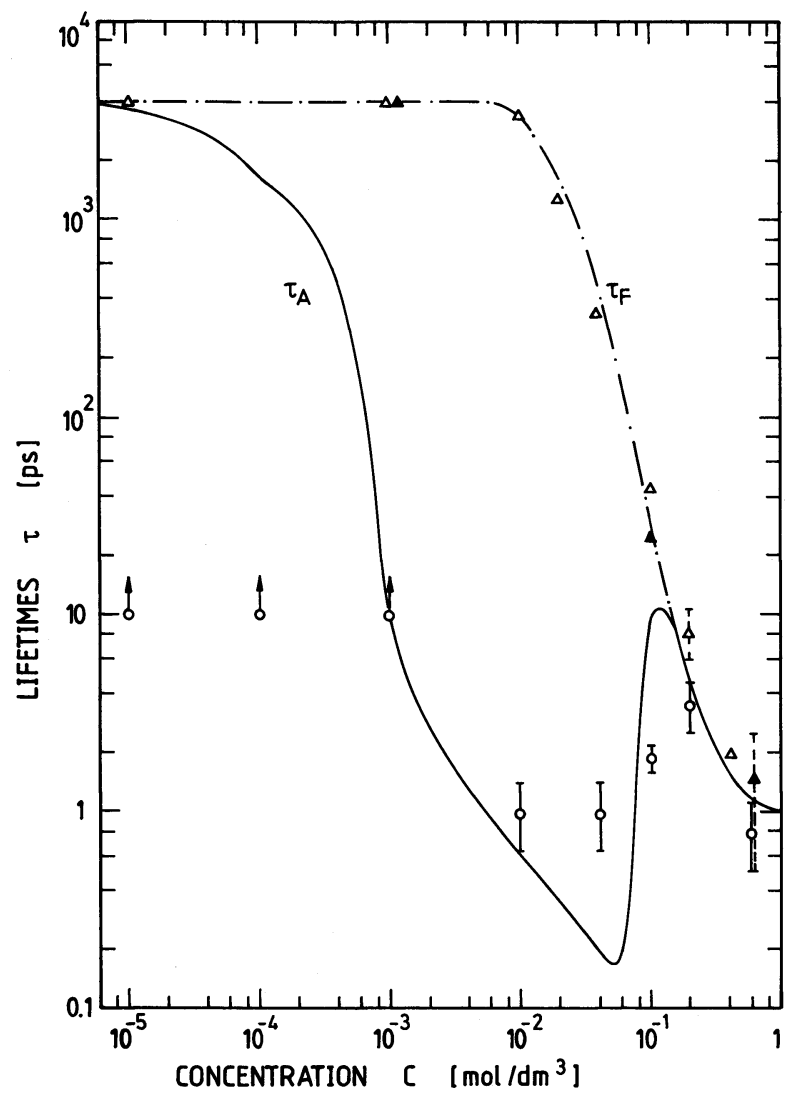

Fig. 6. Dependence of lifetimes on concentration. Experimental points: $(\Delta)$ Fluorescence lifetimes $\tau_{F}$ measured with streak camera [22], $(\Delta) \tau_{F}$ determined by fluorescence quantum efficiency measurements [22] (weak excitation intensity in fluorescence investigations). ( $(0)$ Ground-state absorption recovery times $\tau_{A}$ determined by intensity dependent bleaching of groundstate absorption (points at $10^{-2}, 4 \times 10^{-2}$, and $10^{-2} \mathrm{~mol} / \mathrm{dm}^{3}$ belong to $I_{0 L}=3 \times 10^{9} \mathrm{~W} / \mathrm{cm}^{2}$ ). Curves: $\tau_{F}$ curve, taken from [22], $\tau_{A}$ curve, calculated by use of ( 9 ) including low intensity $S_{1}$ state lifetime $\tau_{F}$ and intensity dependent $S_{1}$-state lifetime shortening due to longitudinal and transverse amplified spontaneous emission. The $\tau_{A}$ curve belongs to $T_{0}=0.01$, $T=0.5 \times \exp \left(-\sigma_{\mathrm{ex}, F} N_{3} l\right)$, and $d_{0}=1 \mathrm{~mm}$
The rise of transmission (probe pulse before the pump pulse) and the decrease of transmission (probe pulse behind the pump pulse) have the same shape. From this time behavior an upper limit of $\tau_{A}<4$ ps could be estimated.

In Fig. 6 the obtained absorption recovery times $\tau_{A}$ are compared with the fluorescence lifetimes $\tau_{F}$ measured under low excitation intensity conditions. The fluorescence lifetimes were determined by streak camera (open triangles) and fluorescence quantum efficiency (closed triangles) measurements [22]. The $\tau_{F}$ points and the $\tau_{F}$ curve are taken from [22]. The steep decay of the fluorescence lifetime above $10^{-2} \mathrm{~mol} / \mathrm{dm}^{3}$ is explained in [22] to be due to unbound dimer formation. The dimers have a very short $S_{1}$-state lifetime $(\approx 1 \mathrm{ps})$ and excited monomers transfer their energy to the dimers. For $C \leqq 10^{-3} \mathrm{~mol} / \mathrm{dm}^{3}$ only lower limits of $\tau_{A}$ could be determined from the energy transmission measurements $(\hat{o})$. In the region between $C=10^{-2}$ and $0.1 \mathrm{~mol} / \mathrm{dm}^{3}$ the absorption recovery times are intensity dependent. The depicted points $(O)$ belong to $I_{0 L} \simeq 3 \times 10^{9} \mathrm{~W} / \mathrm{cm}^{2}$. The absorption recovery time at $0.01 \mathrm{~mol} / \mathrm{dm}^{3}$ is three and a half orders of magnitude shorter than the fluorescence lifetime. For the concentrations 0.2 and $0.6 \mathrm{~mol} / \mathrm{dm}^{3}$ the absorption recovery times $\tau_{A}$ and the fluorescence lifetimes $\tau_{F}$ are the same within the experimental accuracy. The observed intensity dependent deviation of the absorption recovery time from the fluorescence lifetime which was measured under low intensity conditions is explained in the following by including longitudinal and transverse amplified spontaneous emission in the theoretical description.

\section{Effects of Amplified Spontaneous Emission}

In the nonlinear transmission measurements the $S_{1}$ state of the molecules is strongly populated at high pump intensities. For the Stokes shifted fluorescence emission the system is inverted and the spontaneous emission is amplified by stimulated emission [26]. Fluorescence emission occurs in all directions and the amplification acts in all directions. The amplification of spontaneous emission dominates in directions of longest interaction length.

At low concentrations long samples have to be used to achieve a fixed small signal transmission $T_{0}$. For example, at $10^{-5} \mathrm{~mol} / \mathrm{dm}^{3}$ and $T_{0}=0.01$ then length is $l=2 \mathrm{~cm}$ for rhodamine $6 \mathrm{G}$ in methanol at $\lambda_{L}=526.5 \mathrm{~nm}$. Under these conditions the laser beam diameter $d_{0}$ (FWHM) is small compared to the longitudinal interaction length $l_{I, l o}$, which is given by the shorter of either sample length $l$ or the penetration depth $l_{\text {eff }}$ (reduction of input light to $1 / \mathrm{e}$ value) $\left[l_{I . l o}=\min \left(l, l_{\text {eff }}\right)\right]$. The amplified spontaneous emis- 


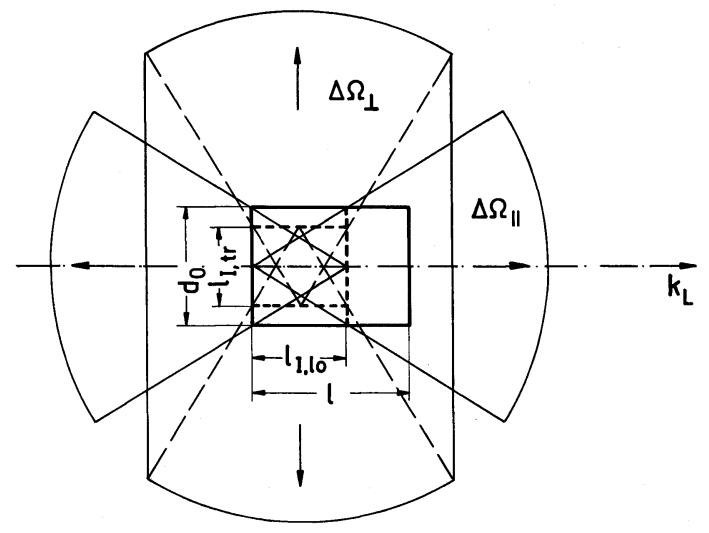

Fig. 7. Illustration of solid angles for longitudinal and transverse amplified spontaneous emission

sion dominates within a solid angle

$\Delta \Omega_{\|} \simeq \frac{\pi d_{0}^{2}}{4 l_{I, l o}^{2}}$

along the propagation direction of the pump beam. This situation is called longitudinal amplified spontaneous emission. Besides the forward direction the longitudinal amplified spontaneous emission occurs in backward direction. As long as the duration of the amplified spontaneous emission is long compared to the propagation time of light through the sample $\left(t_{p, l o}\right.$ $=n l_{I, l_{o}} / c_{0}, n$ : refractive index, $c_{0}$ : vacuum light velocity) forward and backward amplified spontaneous emission are of the same strength. For picosecond pulse excitation and strong amplified spontaneous emission (i.e., drastic $S_{1}$-state lifetime shortening by amplified spontaneous emission) in long cells, the duration of the fluorescence signal becomes short compared to the propagation time $t_{p, l o}$ and backward amplified spontaneous becomes small compared to forward amplified spontaneous emission [27]. In the following estimates backward amplified spontaneous emission is only included for short sample lengths $\left(\Delta \Omega_{\|}>2 \pi\right)$.

With increasing dye concentration the sample length reduces. As soon as the beam diameter $d_{0}$ becomes larger than the longitudinal interaction length, the amplified spontaneous emission dominates in a rotational symmetric section transverse to the pump pulse propagation direction. One speaks of transverse amplified spontaneous emission. The solid angle $\Delta \Omega_{\perp}$ of the transverse amplified spontaneous emission is determined approximately by a cylinder surface of length $l_{I, l_{o}}$ and radius $l_{I, t r}$, that is

$\Delta \Omega_{\perp} \simeq 2 \pi l_{I, t r} l_{I, l o} / l_{I, t r}^{2}=2 \pi l_{I, l o} / l_{I, t r}$.

The maximum transverse interaction length $l_{I, t r}$ is approximately given by the beam diameter $d_{0}$. If the process of amplified spontaneous emission reduces the

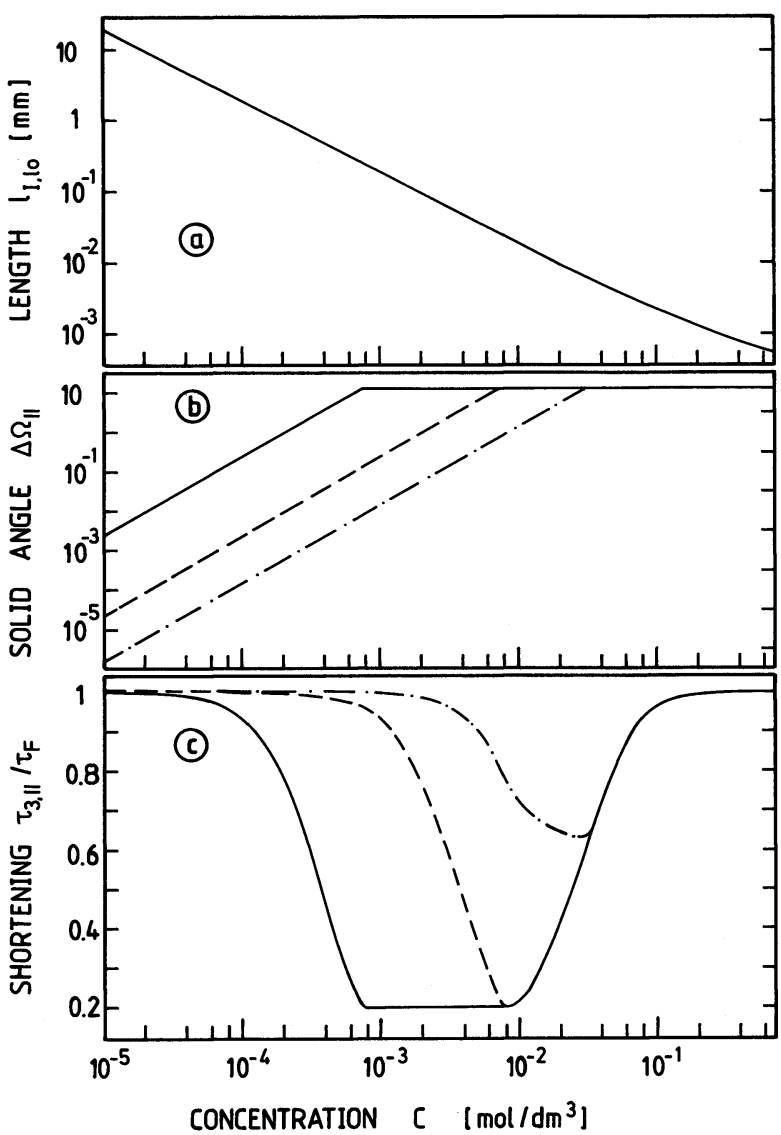

Fig. 8a-c. Longitudinal amplified spontaneous emission. Curves belong to $T_{0}=0.01$ and $T=0.5 \exp \left(-\sigma_{\mathrm{ex}, F} N_{3} l\right)$. (a) Longitudinal interaction length $l_{I, l o}$. It is identical to sample length $l$. (b) Solid angle $\Delta \Omega_{\|}$for beam diameters. $d_{0}=1 \mathrm{~mm}$ (solid curve), $0.1 \mathrm{~mm}$ (dashed curve), and $0.25 \mu \mathrm{m}$ (dash-dotted curve). (c) Shortening of $S_{1}$-state lifetime $\tau_{3, \|} / \tau_{F}$ due to longitudinal amplified spontaneous emission. Dye parameters as in Figs. 2 and 5. Refractive indices $n$ from [24]. Solid curve, $d_{0}=1 \mathrm{~mm}$, dashed curve, $d_{0}=0.1 \mathrm{~mm}$, dash-dotted curve, $d_{0}=25 \mu \mathrm{m}$

$S_{1}$-state lifetime $\tau_{3}$ (equal to $\tau_{A}$ ) to a value shorter than the propagation time $t_{p, t r}=n d_{0} / c_{0}$ across the beam diameter then the transverse interaction length is limited to $l_{I, t r}=l_{p} \simeq \tau_{3} c_{0} / n$. At very high concentration $C \geqq 0.1 \mathrm{~mol} / \mathrm{dm}^{3}$ the longitudinal interaction length $l_{I, l o}$ becomes comparable and shorter than the pump laser wavelength (Fig. $8 \mathrm{a}, l=0.49 \mu \mathrm{m}$ for $C=0.6 \mathrm{~mol} / \mathrm{dm}^{3}$ ). Under these conditions the transverse interaction length reduces to the diffraction length $l_{D}=l_{I, l o} / \sin \theta_{D} \simeq l_{I, l_{o}}^{2} n / \lambda_{L}$. The relevant transverse interaction length is $l_{I, t r}=\min \left(d_{0}, l_{p}, l_{D}\right)$.

An illustration of the longitudinal and transverse solid angles of amplified spontaneous emission is given in Fig. 7. The longitudinal and transverse interaction lengths, $l_{I, l o}$ and $l_{I, t r}$, together with the sample length $l$ and beam diameter $d_{0}$ are indicated.

A theoretical analysis of the longitudinal forward amplified spontaneous emission is given in [27]. 
Extension of the theory of pump pulse propagation under conditions of longitudinal and transverse amplified spontaneous emission leads to a very time consuming computer program. Here were restrict to estimate the absorption recovery time under conditions of longitudinal and transverse amplified spontaneous emission (shortening of $S_{1}$-state lifetime) from a crude analytical analysis [27, 28].

The depopulation of the $S_{1}$ state (level 3 of Fig. 4) after passage of the pump pulse is considered (time region $t>t_{0} \simeq \Delta t_{L} / 2$ ). The population density $N_{3}\left[\mathrm{~cm}^{-3}\right]$ decays due to radiative and radiationless relaxation [time constant $\tau_{F}$, first term of (3)] and amplified

$$
\left.\tau_{3}=\frac{\tau_{F}}{1+\frac{\sigma_{\mathrm{em}} \Delta \Omega \tau_{F}}{4 \pi\left(\sigma_{\mathrm{em}}-\sigma_{\mathrm{ex}, F}\right) \tau_{\mathrm{rad}}}\left[\left(\frac{T \exp \left[\sigma_{\mathrm{ex}, F} N_{3}\left(t_{0}, l_{I} / 2\right) l\right]}{T_{0}}\right)^{\left(\frac{\left.\sigma_{\mathrm{em}}-\sigma_{\mathrm{ex}, F}\right) \frac{l_{I}}{l}}{\sigma_{L}}\right.}-1\right]}\right]^{\cdot}
$$

spontaneous emission [second term of (3)]:

$\frac{\partial}{\partial t^{\prime}} N_{3}\left(z^{\prime}, t^{\prime}\right)=-\frac{N_{3}}{\tau_{F}}-\frac{1}{h v_{\mathrm{F}}} \sigma_{\mathrm{em}} I_{F} N_{3}$.

The propagation of the fluorescence signal is given by

$\frac{\partial}{\partial z^{\prime}} I_{F}=\left(\sigma_{\mathrm{em}}-\sigma_{\mathrm{ex}, F}\right) N_{3} I_{F}+\frac{h v_{F} N_{3} \Delta \Omega}{4 \pi \tau_{\mathrm{rad}}}$.

The transformations $t^{\prime}=t-(n / c) z$ and $z^{\prime}=z$ are used. $\tau_{\text {rad }}$ is the radiative lifetime $\left(\tau_{F}=q_{F} \tau_{\text {rad }}, q_{F}\right.$ : fluorescence quantum efficiency, $\tau_{\mathrm{rad}}=4.3 \mathrm{~ns}$ for rhodamine $6 \mathrm{G}$ in methanol [20]). In (3 and 4) the filling of level 6 by amplified spontaneous emission is neglected $\left(N_{6} \ll N_{3}, \tau_{v} \rightarrow 0\right)$.

Integration of ( 3 and 4$)$ leads to

$$
\begin{aligned}
N_{3}\left(t^{\prime}, z^{\prime}\right)= & N_{3}\left(t_{0}, z^{\prime}\right) \\
& \times \exp \left[-\frac{t^{\prime}-t_{0}}{\tau_{F}}-\frac{\sigma_{\mathrm{em}}}{h v_{F}} \int_{t_{0}}^{t^{\prime}} I_{F}\left(t, z^{\prime}\right) d t\right], \\
I_{F}\left(t^{\prime}, z^{\prime}\right)= & \frac{h v_{F} \Delta \Omega}{4 \pi \tau_{\mathrm{rad}}\left(\sigma_{\mathrm{em}}-\sigma_{\mathrm{ex}, F}\right)} \\
& \times\left\{\exp \left[\left(\sigma_{\mathrm{em}}-\sigma_{\mathrm{ex}, F}\right) N_{3}(\bar{t}, \bar{z}) z^{\prime}\right]-1\right\} .
\end{aligned}
$$

In (6) $t_{0}<\bar{t}<t^{\prime}$ and $0<\bar{z}<z^{\prime}$. Rewriting (5) to $N_{3}\left(t^{\prime}, z^{\prime}\right)$ $=N\left(t_{0}, z^{\prime}\right) \times \exp \left[-\left(t^{\prime}-t_{0}\right) / \tau_{3}\left(t^{\prime}, z^{\prime}\right)\right]$ determines the $S_{1}$-state lifetime to

$$
\begin{aligned}
\tau_{3}\left(t^{\prime}, z^{\prime}\right) & =\left[\frac{1}{\tau_{F}}+\frac{\sigma_{\mathrm{em}}}{h v_{F}\left(t^{\prime}-t_{0}\right)} \int_{t_{0}}^{t^{\prime}} I_{F}\left(t, z^{\prime}\right) d t\right]^{-1} \\
& =\left[\frac{1}{\tau_{F}}+\frac{\sigma_{\mathrm{em}}}{h v_{F}} I_{F}\left(\tilde{t}, z^{\prime}\right)\right]^{-1}
\end{aligned}
$$

where $t_{0}<\tilde{t}<t^{\prime}$. Insertion of (6) into (7) results in with $t_{0} \leqq \tilde{t}<\tilde{t}$ and $0<\tilde{z}<z^{\prime}$. We want to know the momentary decay time of the $S_{1}$-state towards the end of the pump pulse $\left(t^{\prime} \simeq t_{0}\right)$ and at the end of the interaction length of amplified spontaneous emission $\left(z^{\prime}=l_{I}\right)$ and define $\tau_{3}=\tau_{3}\left(t_{0}, l_{I}\right)$. Approximate values for $\tilde{\tilde{t}}$ and $\tilde{\tilde{z}}$ are $\tilde{\tilde{t}} \simeq t_{0}$ and $\tilde{z} \simeq l_{I} / 2$. Using the relations

$N_{3}\left(t_{0}, l_{I} / 2\right)=N_{0}-N_{1}\left(t_{0}, l_{I} / 2\right)$,

$T_{0}=\exp \left(-\sigma_{L} N_{0} l\right)$

and

$T \simeq \exp \left[-\sigma_{L} N_{1}\left(t_{0}, l_{I} / 2\right) l-\sigma_{\text {ex }, F} N_{3}\left(t_{0}, l_{I} / 2\right) l\right]$

leads after some rearrangement to

$N_{0}$ is the total number density of dye molecules. $N_{1}\left(t_{0}, l_{I} / 2\right)$ is the average population density of the $S_{0}$ state towards the end of the pump pulse. $T$ is the pump light transmission at input peak intensity $I_{0 L}$ and $T_{0}$ is the small signal pump light transmission. Equation (9) applies to longitudinal and transverse amplified spontaneous emission. In the longitudinal case it is $\Delta \Omega=\Delta \Omega_{\|},(1)$, and $l_{I}=l_{I, l o}$ while for the transverse case it is $\Delta \Omega=\Delta \Omega_{\perp},(2)$, and $l_{I}=l_{I, t r}$.

Generally, fast saturable absorbers with low fluorescence quantum efficiency $\left(q_{F}=\tau_{F} / \tau_{\mathrm{rad}} \ll 1\right)$ at low concentrations are applied in long cells $\left(d_{0} / l \ll 1\right.$, $\left.\Delta \Omega_{\|} \ll 1\right)$ and the excitation wavelength is around the $S_{0}-S_{1}$ absorption maximum $\left[\left(\sigma_{\mathrm{em}}-\sigma_{\mathrm{ex}, F}\right) / \sigma_{L}<1\right]$. Under these conditions the longitudinal and transverse amplified spontaneous emission are negligibly small. But in the here discussed situation of rhodamine 6G in methanol the fluorescence quantum efficiency varies from $q_{F}=\tau_{F} / \tau_{\mathrm{rad}} \simeq 0.9$ at low concentration to $q_{F}=2.25 \times 10^{-4}$ at high concentration and the cell thickness varies from some centimeters at low concentration to a fraction of a micrometer at high concentration. In this case the actual influence of amplified spontaneous emission depends on the specific dye and laser parameters.

To illustrate the effect of amplified spontaneous emission some calculations are presented in Figs. 6 and $8-10$. A small signal dye transmission of $T_{0}=0.01$ is assumed. The cross-sections $\sigma_{L}, \sigma_{\mathrm{em}}$, and $\sigma_{\mathrm{ex}, F}$ are taken from Fig. 5. $\sigma_{L}$ was measured in [24]. The stimulated emission cross-section $\sigma_{\mathrm{em}}$ in the frequency region of amplified spontaneous emission is approximated by $\sigma_{\mathrm{em}}=0.5 \times \sigma_{L}[29] . \sigma_{\mathrm{ex}, F}$ is set equal to $\sigma_{\mathrm{ex}, L}$

$$
\tau_{3}\left(t^{\prime}, z^{\prime}\right)=\frac{\tau_{F}}{1+\frac{\sigma_{\mathrm{em}} \Delta \Omega \tau_{F}}{4 \pi\left(\sigma_{\mathrm{em}}-\sigma_{\mathrm{ex}, F}\right) \tau_{\mathrm{rad}}}\left\{\exp \left[\left(\sigma_{\mathrm{em}}-\sigma_{\mathrm{ex}, F}\right) N_{3}(\tilde{t}, \tilde{z}) z^{\prime}\right]-1\right\}}
$$




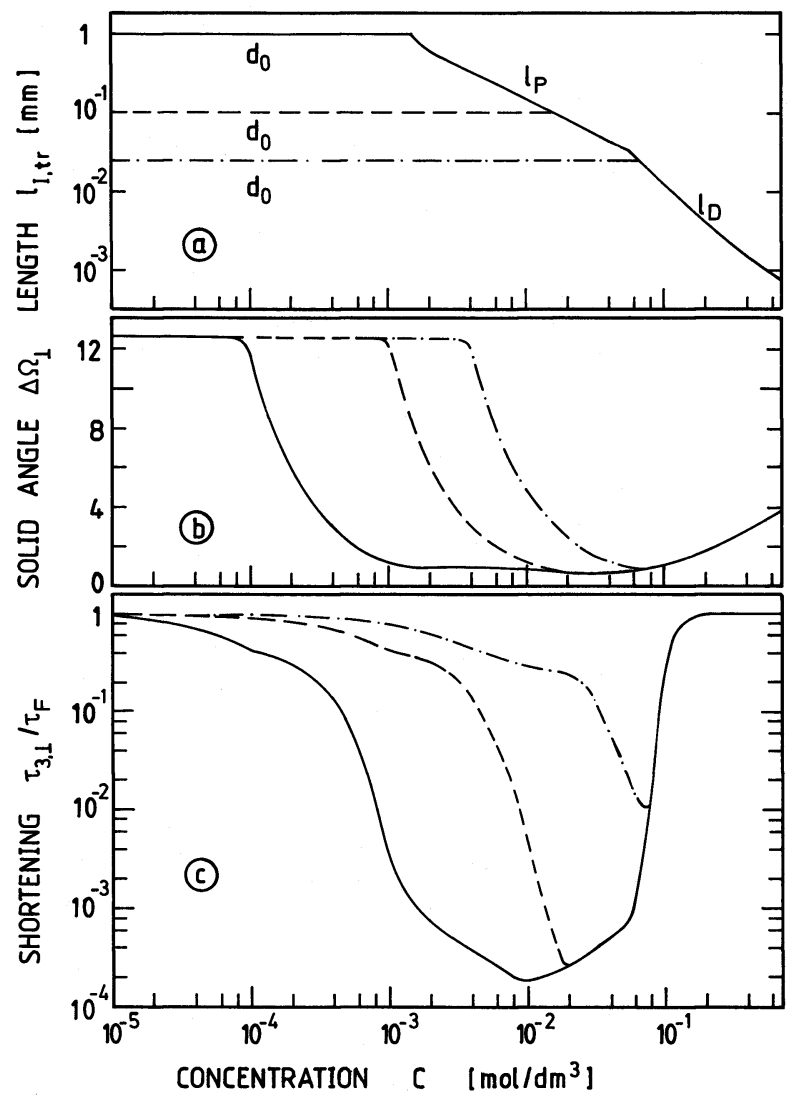

Fig. 9a-c. Transverse amplified spontaneous emission. Curves belong to $T_{0}=0.01$ and $T=0.5 \exp \left(-\sigma_{\mathrm{ex}, F} N_{3} l\right)$. (a) Transverse interaction length $l_{I, t r}$ (b) Solid angle $\Delta \Omega_{\perp}$ for three different beam diameters. $d_{0}=1 \mathrm{~mm}$ (solid curve), $0.1 \mathrm{~mm}$ (dashed curve), and $25 \mu \mathrm{m}$ (dash-dotted curve). (c) Shortening of $S_{1}$-state lifetime $\tau_{3, \perp} / \tau_{F}$ due to transverse amplified spontaneous emission. Dye parameters as in Fig. 8. Beam diameters as in (b)

[25]. The fluorescence lifetime $\tau_{F}$ is included in Fig. 6 $\left(\tau_{\text {rad }} \simeq 4.3 \mathrm{~ns}\right)$.

Figure 8 illustrates the longitudinal amplified spontaneous emission. The bleached transmission is set to

$T=0.5 \exp \left[-\sigma_{\mathrm{ex}, F} N_{3}\left(t_{0}, l_{I} / 2\right) l\right]$.

In Fig. 8a the longitudinal interaction length $l_{I, l o}$ $=\min \left(l, l_{\text {eff }}\right)$ is shown. It is equal to $l$. The solid angle $\Delta \Omega_{\|},(1)$, is depicted in Fig. $8 \mathrm{~b}$ for three different beam diameters $d_{0}=1 \mathrm{~mm}, 0.1 \mathrm{~mm}$, and $25 \mu \mathrm{m}$. The shortening of the $S_{1}$-state lifetime $\tau_{3, \|} / \tau_{F}$ is given by the curves in Fig. 8c. At low concentration (small $\Delta \Omega_{\|}$) the $S_{1^{-}}$ state lifetime shortening is small. In an intermediate concentration range ( $\Delta \Omega_{\|}$large, $\tau_{F}$ large) the lifetime shortening is maximal. At very high concentrations $\tau_{F}$ becomes short and the lifetime shortening by amplified spontaneous emission reduces.

The situation of transverse amplified spontaneous emission is displayed in Fig. 9 for

$T=0.5 \exp \left[-\sigma_{\mathrm{ex}, F} N_{3}\left(t_{0}, l_{I} / 2\right) l\right]$.
Three different beam diameters $d_{0}=1 \mathrm{~mm}, 0.1 \mathrm{~mm}$, and $25 \mu \mathrm{m}$ are considered. The transverse interaction lengths $l_{I, t r}=\min \left(d_{0}, l_{p}, l_{D}\right)$ are shown in Fig. 9a. At low concentrations it is $l_{I, t r}=d_{0}$. In an intermediate concentration region the pulse shortening is so strong that the interaction length is limited by the transverse propagation distance $l_{p}$. At high concentrations the interaction length is limited by diffraction $\left(l_{I, t r}=l_{D}\right)$. The concentration dependence of the solid angle $\Delta \Omega_{\perp}$ is depicted in Fig. $9 \mathrm{~b}$. At low concentrations $\Delta \Omega_{\perp}$ is large $\left(l_{I, t r}<l_{I, l o}\right)$. In an intermediate concentration range $\Delta \Omega_{\perp}$ reduces $\left(l_{I, t r}>l_{I, l o}\right)$. At high concentrations $\Delta \Omega_{\perp}$ increases again due to diffraction effects $\left(l_{I, t r}=l_{D}\right)$. In Fig. 9c the lifetime shortenings $\tau_{3, \perp} / \tau_{F}$ are shown. At low concentrations the shortening is weak. The variation of $\tau_{3, \perp} / \tau_{F}$ with concentration depends strongly on the beam diameter. The larger $d_{0}$ the lower is the necessary concentration for effective $S_{1}$-state depopulation (condition $l_{I, t r}>l_{I, l o}$ fulfilled at lower concentration). In an intermediate concentration region $\left(d_{0}>l_{I, l o}\right)$ the shortening is so large that the transverse propagation distance within $\tau_{3, \perp}$ becomes shorter than $d_{0}$ and

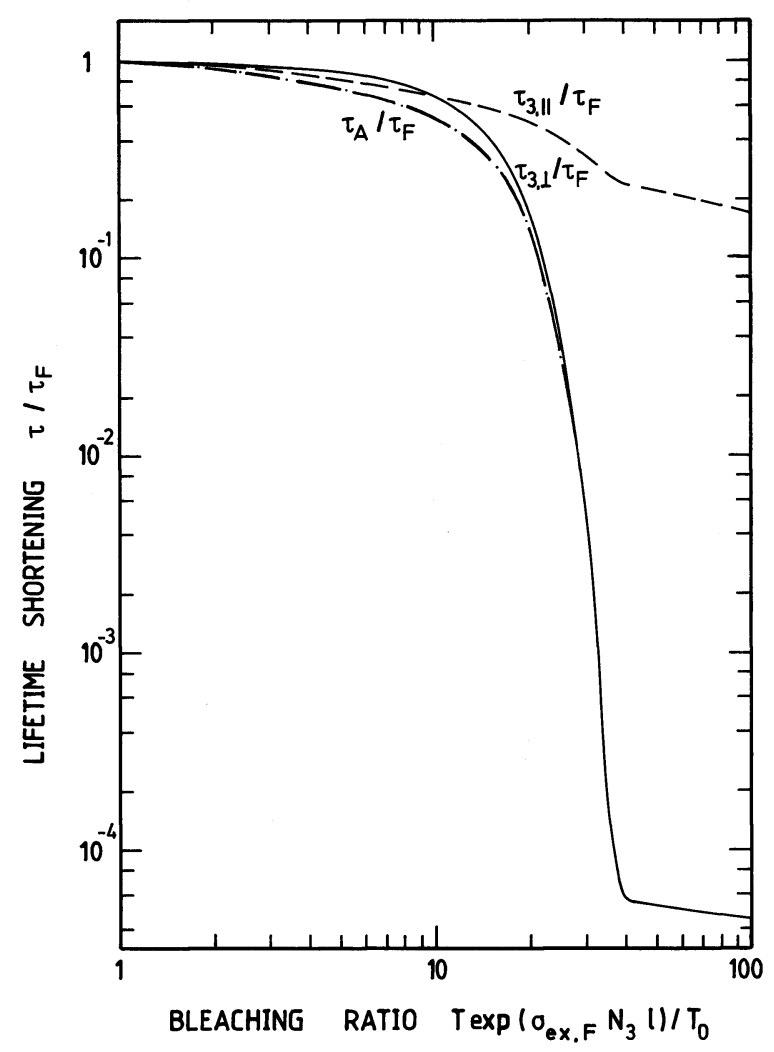

Fig. 10. Intensity dependence of $S_{1}$-state lifetime. The lifetime shortenings $\tau_{3, \|} / \tau_{F}, \tau_{3, \perp} / \tau_{F}$, and $\tau_{A} / \tau_{F}=\left(\tau_{F} / \tau_{3, \|}+\tau_{F} / \tau_{3, \perp}-1\right)^{-1}$ are plotted versus the bleaching ratio $T \exp \left(\sigma_{\mathrm{ex}, F} N_{3} l\right) / T_{0}$. $T_{0}=0.01 . d_{0}=1 \mathrm{~mm}$. The parameters for $C=0.04 \mathrm{~mol} / \mathrm{dm}^{3}$ are used 
limits the shortening. At high concentrations the transverse interaction length is limited by diffraction and $\tau_{F}$ decreases. The lifetime shortening by amplified spontaneous emission reduces.

In the described model of Fig. 4 the $S_{1}$-state lifetime $\tau_{3}$ is equal to the absorption recovery time $\tau_{A}\left[\tau_{A}=\tau_{3}=\left(l / \tau_{3, \|}+1 / \tau_{3, \perp}-1 / \tau_{F}\right)^{-1}\right]$.

In Fig. 6 a calculated $\tau_{A}$ curve is included for a laser beam diameter of $d_{0}=1 \mathrm{~mm}$ and $T=0.5 \exp \left[-\sigma_{\mathrm{ex}, F} N_{3}\left(t_{0} l_{I} / 2\right) l\right]$.

The curve aggrees reasonably with the $\tau_{A}$ points. The correct description of the low absorption recovery times at $10^{-2} \mathrm{~mol} / \mathrm{dm}^{3}$ and $4 \times 10^{-2} \mathrm{~mol} / \mathrm{dm}^{3}$ and the equality of absorption recovery time and fluorescence lifetime at high concentrations should be noticed. The strong dependence of $\tau_{A}$ on the beam diameter (Fig. 9) gives a possibility to change the absorption recovery time by changing the beam diameter (focusing).

The intensity dependence of the absorption recovery time is illustrated in Fig. 10. The $S_{1}$-state lifetime shortenings $\tau_{3, \|} / \tau_{F}, \tau_{3, \perp} / \tau_{F}$, and $\tau_{A} / \tau_{F}$ versus normalized transmission $T \times \exp \left(\sigma_{\mathrm{ex}, F}, N_{3} l\right) / T_{0}$ is plotted for a concentration of $C=4 \times 10^{-2} \mathrm{~mol} / \mathrm{dm}^{3}$ and a beam diameter of $d_{0}=1 \mathrm{~mm}$. At low intensities $\left(T \rightarrow T_{0}=0.01\right)$ no amplified spontaneous emission occurs. At high intensities (larger $T / T_{0}$ values) the longitudinal and the transverse amplification of spontaneous emission shorten the $S_{1}$-state lifetime. The longitudinal shortening levels off when the penetration length $l_{\text {eff }}$ becomes equal to $l$. The transverse shortening is finally limited by the transverse light propagation time.

The transverse amplified spontaneous emission was studied experimentally for the 0.04 molar rhodamine $6 \mathrm{G}$ solution. The dye was contained in a glass cell and the sideward fluorescence was imaged to a photodetector. Strong emission occured transverse to the direction of pump pulse propagation within an opening angle of about 3 degree. The total light emission within this angle summed up over the circumfirence of emission was estimated to be about $50 \%$ of the input pump light.

\section{Conclusions}

The absorption recovery time of rhodamine $6 \mathrm{G}$ in methanol may be varied between about $3.9 \mathrm{~ns}$ at low concentration to about $1 \mathrm{ps}$ at high concentration. The change of absorption recovery time along concentration is due to intensity independent $S_{1}$-state lifetime quenching by dimer formation and due to intensity dependent $S_{1}$-state lifetime shortening by longitudinal and transverse amplified spontaneous emission. The small signal transmission may be adjusted to any needed value by use of a thin cell of variable thickness. The dye rhodamine $6 \mathrm{G}$ in methanol with variable absorption recovery time may be applied as saturable absorber in mode-locked dye lasers or for pulse shortening of second harmonic light pulses of modelocked Nd-glass and Nd-Yag lasers.

The concentration quenching of the $S_{1}$-state lifetime is a general phenomenon [11, 12] and it should be possible for many dyes to tailor the absorption recovery time to the experimental needs. The use of highly concentrated dyes in thin cells of variable thickness as saturable absorbers may be very interesting in situations where saturable absorbers with fast monomeric absorption recovery time are not available.

In recently reported experiments the occurance of strong transverse amplified spontaneous emission was applied to picosecond-pulse generation [30-33].

Acknowledgements. The author is grateful to Prof. W. Kaiser for helpful discussions. He thanks the "Deutsche Forschungsgemeinschaft" for financial support and the "Rechenzentrum" of the University for disposal of computer time.

\section{References}

1. C.R. Giuliano, L.D. Hess: IEEE J. QE-3, 358 (1967)

2. M. Hercher: Appl. Opt. 6, 947 (1967)

3. J.D. Macomber: J. Appl. Phys. 38, 3525 (1967)

4. M. Hercher, W. Chu, D.L. Stockman: IEEE J. QE-4, 954 (1968)

5. L. Huff, L.G. DeShazer: J. Opt. Soc. Am. 60, 157 (1970)

6. G. Grönninger, A. Penzkofer: Opt. Quant. Electron. 16, 225 (1984)

7. S.L. Shapiro (ed.): Ultrashort Light Pulses, Topics Appl. Phys. 18 (Springer, Berlin, Heidelberg 1977)

8. A. Penzkofer: Opto-Electronics 6, 87 (1974)

9. A. Penzkofer, F. Härtinger, J. Wiedmann: Appl. Phys. B26, $239(1981)$

10. F. Graf, J. Schmidt, A. Penzkofer: Opt. Commun. 54, 176 (1985)

11. Th. Förster: Fluoreszenz Organischer Verbindungen (Vandenhoeck and Ruprecht, Göttingen 1951)

12. C.A. Parker: Photoluminescence of Solutions (Elsevier, Amsterdam 1968)

13. D.A. Cremers, M.W. Windsor: Chem. Phys. Lett. 71, 27 (1980)

14. V.Sundström, T. Gillbro, H. Bergström: Chem. Phys. 73, 439 (1982)

15. W. Siebrand, D.F. William: J. Chem. Phys. 49, 1860 (1968)

16. W. Kranitzky, B. Kopainsky, W. Kaiser, K.H. Drexhage, G.A. Reynolds: Opt. Commun. 36, 149 (1981)

17. B. Kopainsky, P. Qiu, W. Kaiser, B. Sens, K.H. Drexhage: Appl. Phys. B29, 15 (1982)

18. Th. Förster, E. König: Z. Elektrochemie 61, 344 (1957)

19. R.R. Alfano, S.L. Shapiro, W. Yu: Opt. Commun. 7, 191 (1973)

20. K.A. Selanger, J. Falnes, T. Sikkeland: J. Phys. Chem. 81, 1960 (1977) 
21. B. Kopainsky, W. Kaiser: Chem. Phys. Lett. 88, 357 (1982)

22. A. Penzkofer, Y. Lu: Chem. Phys. (1986) (to be published)

23. A. Penzkofer, W. Falkenstein: Opt. Commun. 16, 247 (1976)

24. Y. Lu, A. Penzkofer: Chem. Phys. (to be published)

25. J. Wiedmann, A. Penzkofer: Nuovo Cimento 63B, 459 (1981)

26. M.E. Mack: Appl. Phys. Lett. 15, 166 (1969)

27. A. Penzkofer, W. Falkenstein: Opt. Quant. Electron. 10, 399 (1978)

28. A. Penzkofer, W. Blau: Opt. Quant. Electron. 15, 325 (1983)
29. W. Falkenstein, A. Penzkofer, W. Kaiser: Opt. Commun. 27, 151 (1978)

30. H.J. Polland, T. Elsaesser, A. Seilmeier, W. Kaiser: Appl. Phys. B32, 53 (1983)

31. Zs. Bor, S. Szatmari, A. Müller: Appl. Phys. B32, 101 (1983)

32. T. Elsaesser, H.J. Polland, A. Seilmeier, W. Kaiser: IEEE J. QE-20, 191 (1984)

33. S. Szatmari, F.P. Schäfer: Opt. Commun. 49, 281 (1984)

34. H.E. Lessing, A. von Jena: In: Laser Handbook, Vol. 3, ed. by M.E. Stitch (North-Holland, Amsterdam 1979) p. 821 